\title{
Roboto Pi: uma Plataforma Robótica para o Ensino de Introdução à Programação com Python
}

\author{
Robson S. Lima ${ }^{1}$, Josué da Silva Gomes Júnior ${ }^{2}$, Alisson V. de Brito ${ }^{2}$, Ana Liz S. O. Araújo ${ }^{1}$ \\ ${ }^{1}$ Centro de Ciências Aplicadas e Educação - Universidade Federal da Paraíba (UFPB) \\ Caixa Postal: 58297-000 - Rio Tinto - PB - Brasil \\ ${ }^{2}$ Centro de Informática - Universidade Federal da Paraíba (UFPB) \\ Caixa Postal: 58058-600 - João Pessoa - PB - Brasil \\ \{robson.soares, josue.gomes, analiz\}@dcx.ufpb.br, alisson@ci.ufpb.br
}

\begin{abstract}
Introductory programming classes have high evasion and failure rate. To minimize this problem, the research proposes to introduce robotics in the classroom. However, there are few robotics kits available to undergraduate. Thus, this paper aims to present the development of a pedagogical robotics kit, called Roboto Pi, intended for teaching Introductory programming classes for undergraduate. The Roboto Pi does not require prior knowledge of basic electronics. Besides, it implements a Python API for programming the sensors.
\end{abstract}

Resumo. O ensino de programação introdutória em cursos técnicos e superiores possui problemas de alta taxa de evasão e reprovação. Para minimizar esse problema, pesquisas propõem introduzir a robótica pedagógica $(R P)$ em sala de aula. Entretanto, identificamos que há poucos kits no mercado que visam atender esse público. Assim, esse artigo tem o objetivo de apresentar o desenvolvimento de um kit de RP, chamado Roboto Pi, projetado para auxiliar o ensino de introdução à programação para alunos de cursos técnicos e superiores na área de Computação. O kit de RP Roboto Pi não exige que os alunos tenham conhecimento prévio em eletrônica básica. Além disso, ele implementa uma API Python para programação dos sensores.

\section{Introdução}

As disciplinas introdutórias de programação passam por problemas de alta taxa de evasão e reprovação no ensino superior [Giraffa e Mora 2016]. O ensino de programação tem o objetivo de proporcionar aos alunos o desenvolvimento de suas capacidades de resolução de problemas com o auxílio de linguagem de programação [Gomes et al. 2008]. Os ingressantes nos cursos técnicos e superiores de Computação sentem dificuldades nas disciplinas introdutórias de programação, o que acarreta a desmotivação logo no início do curso. Além disso, [Gomes et al. 2012] enfatiza que os alunos manifestam preferência por aulas com maior componente prático, em detrimento de uma exposição de conteúdos excessiva.

Segundo [Zanetti e Oliveira 2016], é necessário criar um ambiente diversificado e motivador para o aluno utilizando a Robótica Pedagógica (RP), pois a RP promove um vasto conjunto de recursos para promover práticas estimulantes. A RP vem sendo amplamente utilizada no ensino médio e técnico como artefato cognitivo despertando o interesse dos jovens pela robótica e programação. 
VII Congresso Brasileiro de Informática na Educação (CBIE 2018)

Anais do XXIX Simpósio Brasileiro de Informática na Educação (SBIE 2018)

No mercado existem diversas opções de kits de robótica para serem usados como ferramentas de ensino-aprendizagem de programação, mas que possuem altos custos, o que inviabiliza a aquisição em alguns casos. Além disso, na robótica, alguns componentes necessitam que o usuário possua um prévio conhecimento em eletrônica básica. Esses conhecimentos incluem desde conceitos básicos como tensão, resistência e capacitância, até a protocolos de comunicação dos sensores. Esses conhecimentos básicos, somado aos altos custos dos kits comerciais, podem desmotivar os alunos e professores a utilizarem a RP como um meio para ensinar programação.

É importante uma plataforma de RP que supra as necessidades dos professores em relação aos conteúdos ministrados na disciplina de introdução à programação, que seja de fácil utilização e que tenha como foco principal o estudo de algoritmos e linguagem de programação para que possa ser introduzido sem afetar o fluxograma do curso. Diante desse contexto, esse artigo tem o objetivo de apresentar o desenvolvimento de um kit de robótica pedagógico chamado Roboto Pi, destinado ao ensino de introdução à programação para alunos de cursos técnicos e superiores na área de Computação. $\mathrm{O}$ kit de RP Roboto Pi não exige que os alunos tenham conhecimento prévio em eletrônica básica. Além disso, ele implementa uma solução de software que facilita a utilização dos sensores empregando a linguagem de programação Python. Python foi a linguagem escolhida porque possui diversas vantagens em relação a outras linguagens, como portabilidade, sintaxe clara, tipagem dinâmica, multiparadigma, extensa biblioteca de funções e feedback imediato [Miller 2004].

O artigo está organizado da seguinte forma: na seção 2 apresentamos uma breve fundamentação teórica sobre a utilização de RP. Em seguida, na seção 3, discutimos trabalhos relacionados. Na seção 4, descrevemos a metodologia de desenvolvimento do Roboto Pi. Na seção 5, apresentamos resultados. Finalmente, na seção 6 apresenta as considerações finais e trabalhos futuros.

\section{Fundamentação Teórica}

Para compreender o uso da RP em sala de aula, é necessário compreender as teorias de aprendizagem. Segundo [Rocha 2006], em termos epistemológicos, o conhecimento é uma relação entre o homem, chamado de sujeito, e o mundo, real ou abstrato, chamado de objeto. A relação sujeito-objeto é explanada segundo paradigmas filosóficos, a exemplo do Racionalismo, Empirismo e o Interacionismo.

No racionalismo, é considerado que a razão é a fonte do conhecimento, onde o conhecimento provém do sujeito. Desse modo, considera-se que o homem ao nascer, já possui um pré-conhecimento estabelecido e utiliza das experiências sensoriais para despertar a consciência do conhecimento. [Neves e Damiani 2006] explana em seu trabalho que o professor, na visão empirista, considera que o aluno somente escuta, copia e executa. Além de que o professor dita, ensina e decide o que fazer. Na visão de [Becker 2008], a epistemologia interacionista o professor acredita que só haverá aprendizado se o aluno praticar e problematizar sua ação, esse processo fará com que ele aprenda por meio de experiência e reflexão. [Gomes et al. 2008] menciona que muito do que é lecionado em sala de aula, atualmente, utiliza métodos de estudos baseados em leituras sucessivas, memorização e mecanização de procedimentos.

A RP no âmbito do ensino de programação, traz consigo características da epis- 
VII Congresso Brasileiro de Informática na Educação (CBIE 2018)

Anais do XXIX Simpósio Brasileiro de Informática na Educação (SBIE 2018)

temologia interacionista, saindo do contexto das aulas tradicionais baseadas nas epistemologias racionalistas e empiristas, possibilitando assim criar um ambiente que, além de motivar, proporcione um ambiente colaborativo e reflexivo. A RP é caracterizada pela aprendizagem baseada em sistemas compostos por peças nas quais é possível montar um robô programável. Esses robôs possuem uma estrutura que possibilita a realização de atividades físicas ou de movimento. [Rocha 2006] explica que ao trabalhar em um ambiente de RP, o objeto de programação passa a ser um dispositivo robótico construído ou montado pelos alunos, sendo esse um artefato cognitivo.

\section{Trabalhos Relacionados}

Existem diversos trabalhos na literatura que possuem como objetivo propiciar melhorias no processo de aprendizagem de programação utilizando robótica. Selecionamos dois trabalhos acadêmicos, com base no uso para o ensino de programação através de kits robóticos. [Miranda et al. 2010] apresentam o desenvolvimento de um kit de robótica chamado RoboFácil, de baixo custo para ensinar conceitos básicos de programação. $\mathrm{O}$ software ProgrameFácil foi desenvolvido para o hardware do kit. O objetivo foi criar um material que tivesse uma interface de fácil utilização e programação. O projeto foi testado com simulações de uso em contexto educacional realizadas com alunos de PósGraduação em Informática, e demonstraram ter grande potencial de aplicação e ensino e aprendizagem de introdução à programação.

O trabalho de [Blank et al. 2004] apresenta um ambiente de programação chamado Pyro, projetado para uso da robótica em conjunto com uma linguagem de alto nível, sem que haja a preocupação de que o usuário conheça o projeto eletromecânico. O Pyro tem como objetivo disponibilizar um sistema simples para que alunos iniciantes possam utilizá-lo, trabalhar com um paradigma de programação orientado a objetos e ser multiplataforma. Além disso, o projeto visou possibilitar ao usuário desenvolver diferentes metodologias de controle de robôs. O projeto foi utilizado por estudantes ingressantes em Ciência da Computação em uma disciplina de graduação por um semestre letivo.

Ambos os trabalhos foram propostos para facilitar o ensino de programação e obtiveram bons resultados ao serem aplicados em disciplinas introdutórias de programação, e utilizam linguagem de alto nível para que seja mais fácil a programação dos kits e que haja maior aderência por parte dos alunos. Os dois trabalhos se assemelham ao kit proposto neste trabalho por utilizar o linguagem de alto nível e ser de baixo custo e para o mesmo público.

\section{Metodologia}

Para o desenvolvimento de um kit de RP que pudesse atender as necessidades dos professores e alunos que estão iniciando no estudo de introdução à programação, realizamos os procedimentos descritos a seguir. Iniciamos a pesquisa realizando uma análise dos kits de RP disponíveis no mercado e desenvolvidos na academia, com o propósito de identificar as características presentes e ausentes em kits de RP. Em seguida, descrevemos a especificação de hardware do Roboto Pi, seguido pela especificação de software (Application Programming Interface - API Python) e a produção de manuais para o aluno e para o professor. 
VII Congresso Brasileiro de Informática na Educação (CBIE 2018)

Anais do XXIX Simpósio Brasileiro de Informática na Educação (SBIE 2018)

\subsection{Análise de Kits de Robótica Pedagógica}

A seleção de kits de RP para análise, utilizou como critério a arquitetura, a quantidade e os tipos de recursos e sensores disponíveis para uso, além da linguagem de programação utilizada, bem como a quantidade de referências encontradas sobre cada kit. Dessa forma, para essa análise de recursos foram escolhidos seis kits: LEGO $^{\circledR}$ Mindstorms ${ }^{\circledR}$ EV3, Fischertechnik $^{\circledR}$ ROBO TX Explorer, ALPHA Maker, Hajime, Robofácil e o TESLA.

LEGO $^{\circledR}$ Mindstorms ${ }^{\circledR}$ EV3 é um kit de iniciação à robótica desenvolvida pela empresa LEGO $^{\circledR}$, mundialmente conhecida pela inovação na área de brinquedos, que consiste em, através de peças encaixáveis, o usuário pode montar um robô programável. $\mathrm{O}$ kit pode assumir diversas formas com base na estrutura que o usuário montar a partir das peças disponíveis no kit. O hardware é constituído de um processador com base na arquitetura ARM9 de 32-bits, clock de $300 \mathrm{MHz}$, e $64 \mathrm{MB}$ de RAM. Na parte de softwares, o kit conta com uma versão personalizada do Linux e utiliza uma conexão USB para ser programado utilizando um PC. A programação é feita a partir de blocos o que facilita o aprendizado para o público alvo, estudantes dos ensinos fundamentais e médio.

Fischertechnik $^{\circledR}$ ROBO TX Explorer é um kit desenvolvido pela empresa Fischertechnik ${ }^{\circledR}$ que possui como objetivo o aprendizado de robótica e é voltado para competições. A estrutura é totalmente voltada para a atividade de resgate com seguidor de linha e as peças utilizam o sistema de montagem por encaixe. O controlador possui como processador um ARM Cortex A8 (32bit/500mHz), um co-procesador Cortex M3 (32bit/200mHz) e capacidade de memória de 128 MB DDR3 RAM com 64 MB Flash. O software utilizado para a programação do robô utiliza linguagem em blocos, não sendo suportado nenhuma outra por padrão. Possui conexão e Wifi e Bluetooth usados para conexão remota utilizando um smartphone.

O Kit ALPHA Maker desenvolvido e comercializado nacionalmente pela empresa Pete é composto por uma unidade de controle compatível com a arquitetura de micro controladores Arduino. Em relação a base de suporte ela é feita de plástico feita em impressora 3D assim como as peças. Toda a estrutura é montada utilizando porcas e parafusos disponibilizados. A programação é feita usando software proprietário chamado "LEGAL", que utiliza blocos de comando em português. Um problema que foi observado especificamente por esse kit é a falta de documentação técnica do controlador utilizado, que impossibilita uma comparação com outras soluções disponíveis no mercado.

O TESLA é um kit de robótica educacional de baixo custo voltado para utilização em universidades brasileiras, especificamente nos cursos de engenharia e computação. Conforme [Silva 2017], a plataforma é composta de duas rodas, uma garra articulada, módulo de potência, de controle, de sensores, de controle do braço mecânico e de sensores de luz, montados sobre um chassi de poliuretano. A programação é feita utilizando o software MAPLAB utilizado para gravação de micro controladores da família PIC. O projeto suporta as linguagens Assembly e C podendo com a utilização de softwares de terceiros a utilização de Pascal e Ladder.

A Tabela 1 resume a metodologia de programação utilizada, método de programação icônica ou textual, arquitetura eletrônica, interface de comunicação com um PC, e o valor de cada kit com base nas especificações de seus fabricantes em seus respec- 
VII Congresso Brasileiro de Informática na Educação (CBIE 2018)

Anais do XXIX Simpósio Brasileiro de Informática na Educação (SBIE 2018)

tivos sites. A última coluna da tabela apresenta as informações do Roboto Pi. Os valores foram especificados com base em uma pesquisa de preços em lojas virtuais (Amazon e Mercado Livre) com os valores de impostos já incluídos.

Tabela 1. Análise de critérios em kits.

\begin{tabular}{|c|c|c|c|c|c|c|}
\hline$\stackrel{\text { Kit }}{\text { Caracteristicas }}$ & $\begin{array}{l}\text { Lego EV3 } \\
\text { Mindstorms }\end{array}$ & $\begin{array}{l}\text { Fischertechnik } \\
\text { ROBO TX } \\
\text { Explorer }\end{array}$ & Alpha Maker & Robofácil & TESLA & Roboto Pi \\
\hline Arquitetura & ARM9 de 32 bits & $\begin{array}{l}\text { ARM Cortex A8 de } \\
32 \text { bits }\end{array}$ & Não especificado & Intel MCS-51 & PIC16F84 & $\begin{array}{c}\text { Broadcom BCM2837 } \\
64 \text { bit ARMv8 } \\
\text { Cortex-A53 Quad- } \\
\text { Core }\end{array}$ \\
\hline Open source & Não & Não & Não & Sim & Sim & Sim \\
\hline $\begin{array}{l}\text { Interface com o } \\
\text { computador }\end{array}$ & Sim & Sim & Sim & Sim & Sim & $\mathrm{SSH} / \mathrm{VNC} / \mathrm{USB}$ \\
\hline $\begin{array}{l}\text { Programação por linhas } \\
\text { de comando }\end{array}$ & Não & Não & Não & Sim & $\operatorname{Sim}$ & Sim \\
\hline Programação icônica & Sim & Sim & Sim & Sim & Não & Sim \\
\hline Fabricação & Internacional & Internacional & Nacional & Nacional & Nacional & \\
\hline Peças & Encaixáveis & Encaixáveis & Parafusos & Parafusos & Parafusos & Parafusos \\
\hline Possui câmera & Não & Não & Não & Não & Não & Sim \\
\hline Acelerômetro/giroscópio & Sim & Não & Sim & Não & Não & $\mathrm{Sim}$ \\
\hline $\begin{array}{l}\text { Suporte à reprodução de } \\
\text { mídia }\end{array}$ & Sim & Não & Não & Não & Não & Sim \\
\hline Sensor de temperatura & Não & Não & Não & Não & Não & Sim \\
\hline Foto resistor & Não & Sim & $\mathrm{Sim}$ & Sim & Sim & $\mathrm{Sim}$ \\
\hline Sensor ultrassônico & Sim & Sim & $\mathrm{Sim}$ & $\mathrm{Sim}$ & $\mathrm{Sim}$ & $\mathrm{Sim}$ \\
\hline Sensor infravermelho & Sim & Sim & $\mathrm{Sim}$ & $\mathrm{Sim}$ & Sim & Sim \\
\hline Sensor de cor & Sim & Não & $\mathrm{Sim}$ & $\mathrm{Sim}$ & Não & $\mathrm{Sim}$ \\
\hline Sensor de toque & Sim & Não & $\mathrm{Sim}$ & $\operatorname{Sim}$ & Sim & $\operatorname{Sim}$ \\
\hline Motores & Sim & Sim & Sim & $\mathrm{Sim}$ & $\mathrm{Sim}$ & Sim \\
\hline Display & Sim & $\operatorname{Sim}$ & $\mathrm{Sim}$ & $\mathrm{Sim}$ & Não & $\operatorname{Sim}$ \\
\hline Custo (RS) & 2.500 & 1.493 & 1.500 & - & 300,00 & 709,00 \\
\hline
\end{tabular}

Os kits citados nesta seção e os trabalhos relacionados, apesar de terem objetivos similares, possuem elementos individuais característicos que, quando unidos, formariam um kit de robótica com recursos completos para utilização em aulas de ensino técnico e superior. O desenvolvimento do projeto Roboto Pi implementou as características individuais dos kits pesquisados em uma nova solução completa. Essas características foram o baixo custo, a interface de abstração de conhecimentos de eletrônica, e uma plataforma robótica móvel, diferente do trabalho de [Kato et al. 2015] que possui essa limitação. Outro ponto importante a destacar: nos dois últimos kits não há material documental ou educativo, o que dificulta o uso do kit por terceiros.

\subsection{Descrição do Hardware do Roboto Pi}

O hardware do Roboto Pi foi dividido nos seguintes componentes: estrutura montável (chassi), controlador, interface dos sensores, sensores.

\subsubsection{Estrutura montável (chassi)}

Usando processo de modelagem 3D, criamos um modelo estrutural em 3D (ver Figura 1) que permite o acoplamento de sensores, é compacto, permite fácil manutenção dos componentes e apresenta baixo custo de produção. Esse processo foi feito usando o software Blender 3D, que segundo [Roosendaal 2007], é um software de modelagem e animação 3D de código aberto, com recursos que permitem desenvolver modelos, animações e simulações. O modelo em 3D foi reproduzido usando peças de acrílico, pois esse material se torna acessível para ser produzido em quantidade além de facilitar a reposição das peças. O modelo também pode ser produzido por impressão 3D. Como a plataforma tem como público alvo, alunos que podem manipular peças pequenas, para os encaixes dos componentes, utilizamos parafusos, porcas e espaçadores de Nylon de tamanho M3. 

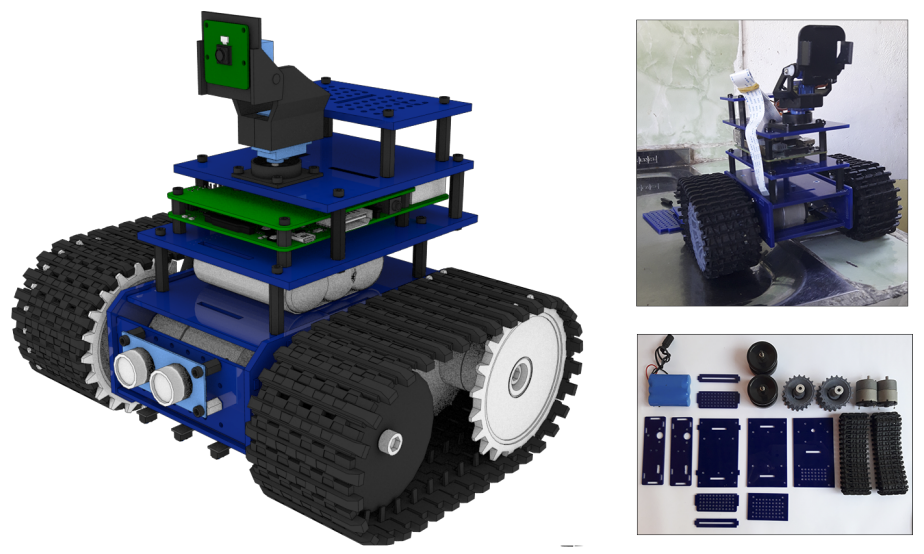

Figura 1. Modelo 3d e estrutura real do kit Roboto Pi.

As dimensões do kit tiveram como base as regras da Olimpíada Brasileira de Robótica ${ }^{1}$ na modalidade "RoboCup Junior Rescue Line". Escolhemos essas dimensões para possibilitar que o Roboto Pi também esteja apto a participar de competições nacionais. Essa modalidade especifica dimensões máxima do robô de até $25 \mathrm{~cm}$ por $25 \mathrm{~cm}$. Desse modo, o Roboto Pi possui como dimensões $20 \mathrm{~cm}$ de altura por $17,5 \mathrm{~cm}$ de largura, dentro dos limites permitidos na competição.

\subsubsection{Controlador}

O controlador possui papel principal de controle dos fluxos de funcionamento do robô. Nele, o usuário programa através de instruções de código de forma de textual, ou utilizando linguagens visuais. Grande parte dos kits de robótica disponíveis no mercado e os analisados neste trabalho utilizam controladores desenvolvidos pelas próprias empresas que as comercializam. Como consequência, cada kit possui sua própria linguagem de programação.

No projeto de definição de componentes do Roboto Pi, optamos por escolher a placa Raspberry Pi, por ser uma placa de baixo custo e completa de recursos de hardware e software. Conforme [Marques et al. 2014] explana, a Raspberry $\mathrm{Pi}^{\circledR}$ foi idealizada com o objetivo de ensinar computação e programação nas escolas possuindo um hardware de baixo custo, dimensões pequenas e baixo consumo de energia. A placa utiliza a característica SoC (System-on-a-chip) que é um chip eletrônico composto por diversos componentes principais utilizados em um sistema computacional, dentre eles: CPU, memória RAM, memória ROM e outros componentes adicionais.

\subsubsection{Interface dos sensores}

Para facilitar a aplicação da plataforma robótica em disciplinas introdutórias de programação, desenvolvemos uma interface de hardware que abstraísse conceitos de eletrônica básica, pois em determinados cursos não faz parte da estrutura curricular. Desse

\footnotetext{
${ }^{1}$ http://www.obr.org.br/modalidade-pratica/etapa-nacional
} 
VII Congresso Brasileiro de Informática na Educação (CBIE 2018)

Anais do XXIX Simpósio Brasileiro de Informática na Educação (SBIE 2018)

modo, o professor não precisará ensinar conteúdo de eletrônica básica, nem alterar o fluxo de conteúdos da disciplina para utilizar a estrutura robótica.

Antes do desenvolvimento da placa de interface a ser conectada ao Raspberry $\mathrm{Pi}$, foi preciso definir quais os sensores estariam disponíveis no kit. Com o auxílio da pesquisa comparativa dos kits disponíveis no mercado, identificamos quais sensores são usualmente utilizados e a ausência de outros não disponíveis. Os componentes foram escolhidos com base na premissa de custo-benefício permitindo que plataforma apresentasse preço mais acessível. Os sensores utilizados são: Sensor Ultrassônico, sensor infravermelho, câmera, termômetro, acelerômetro e giroscópio. O kit robótico descrito custou em valores atuais $\mathrm{R} \$ 709,00$ reais, com os componentes importados de sites chineses, com o dólar em uma cotação de $\mathrm{R} \$ 3,60$.

Uma vez os sensores definidos e com o uso dos datasheets dos componentes, projetamos o esquema elétrico da placa de interface onde todos os sensores são conectados. Utilizamos os programas Fritzing e Kicad (http://kicad-pcb.org/) para auxiliar no projeto, pois são ferramentas opensource para prototipagem e criação de PCBs (Placas de Circuito Impresso).

\subsection{API Python}

A API padrão de controle dos pinos digitais e de comunicação do Raspberry pode ser complexa para quem está começando a aprender programação ou não tem familiaridade com a plataforma. Como o público alvo do Roboto Pi são alunos ingressantes nas disciplinas introdutórias de programação, foi necessário criar uma API para abstrair as bibliotecas padrões de controle do Raspberry Pi. Uma API pode ser compreendida como uma composição de ferramentas ou funções que possuem o objetivo de facilitar a programação de uma aplicação. Dessa forma, o desenvolvimento da API do Roboto Pi tem como objetivo facilitar a utilização dos sensores, possibilitando que o usuário somente necessite instanciar o objeto que correspondente ao sensor e usar seus métodos para obter a informação. Assim, foi criada uma API Python para facilitar a programação no Roboto Pi. Portanto, o professor pode utilizar a API para ensinar programação com a linguagem Python, além de usar o kit de RP.

\subsection{Manuais}

Para auxiliar os alunos na utilização do Raspberry Pi e a API do Roboto Pi, desenvolvemos dois manuais, um para o aluno e outro para o professor, contendo exemplos de uso dos sensores. A partir disso, o manual do aluno foi divido em: montagem da plataforma, descrição dos sensores, exemplos de aplicações, esquema de montagem e exemplo de utilização usando a API. Os exemplos possuem imagens referentes a conexão física dos sensores e o código usando a API para controle ou obtenção de dados. Além disso, permite que seja possível conhecer a teoria de como os sensores funcionam e onde são usados em aplicações do dia a dia.

O manual do professor tem o objetivo de auxiliar professores que não possuem conhecimento em RP, mas que desejam usar em disciplinas de introdução à programação. O manual do professor contém exemplos de atividades sugeridas de utilização dos sensores da plataforma com seus esquemas de conexão e código. Além disso, foi criado planos de aulas, sugeridos para a aplicação de atividades relacionadas aos conteúdos vistos em 
VII Congresso Brasileiro de Informática na Educação (CBIE 2018)

Anais do XXIX Simpósio Brasileiro de Informática na Educação (SBIE 2018)

disciplinas introdutórias de programação. Assim, os cursos que utilizarem o Roboto Pi não precisarão alterar a ementa para a inclusão do uso da robótica.

\section{Resultados}

O Roboto Pi foi desenvolvido contendo uma estrutura em acrílico (chassi) responsável por acoplar o controlador e os sensores a serem utilizados pelos alunos. Posteriormente, criamos um esquema elétrico da interface de hardware para abstrair o conhecimento em eletrônica básica, possibilitando a utilização desses por meio do encaixe dos conectores dos sensores no controlador. Além disso, implementamos uma API em linguagem de programação Python que abstrai a utilização dos sensores, possibilitando uma melhor compreensão em menos linhas de código.

\subsection{Exemplo de código}

O algoritmo 1 mostra um código usando API Python que utiliza sensores (infravermelhos e os motores) para exemplificar o funcionamento de uma estrutura de repetição usando a movimentação. O código mostra como pode ser feito a instanciação dos infravermelhos e dos motores, bem como o funcionamento do comando while, que desliga os motores caso detecte uma linha.

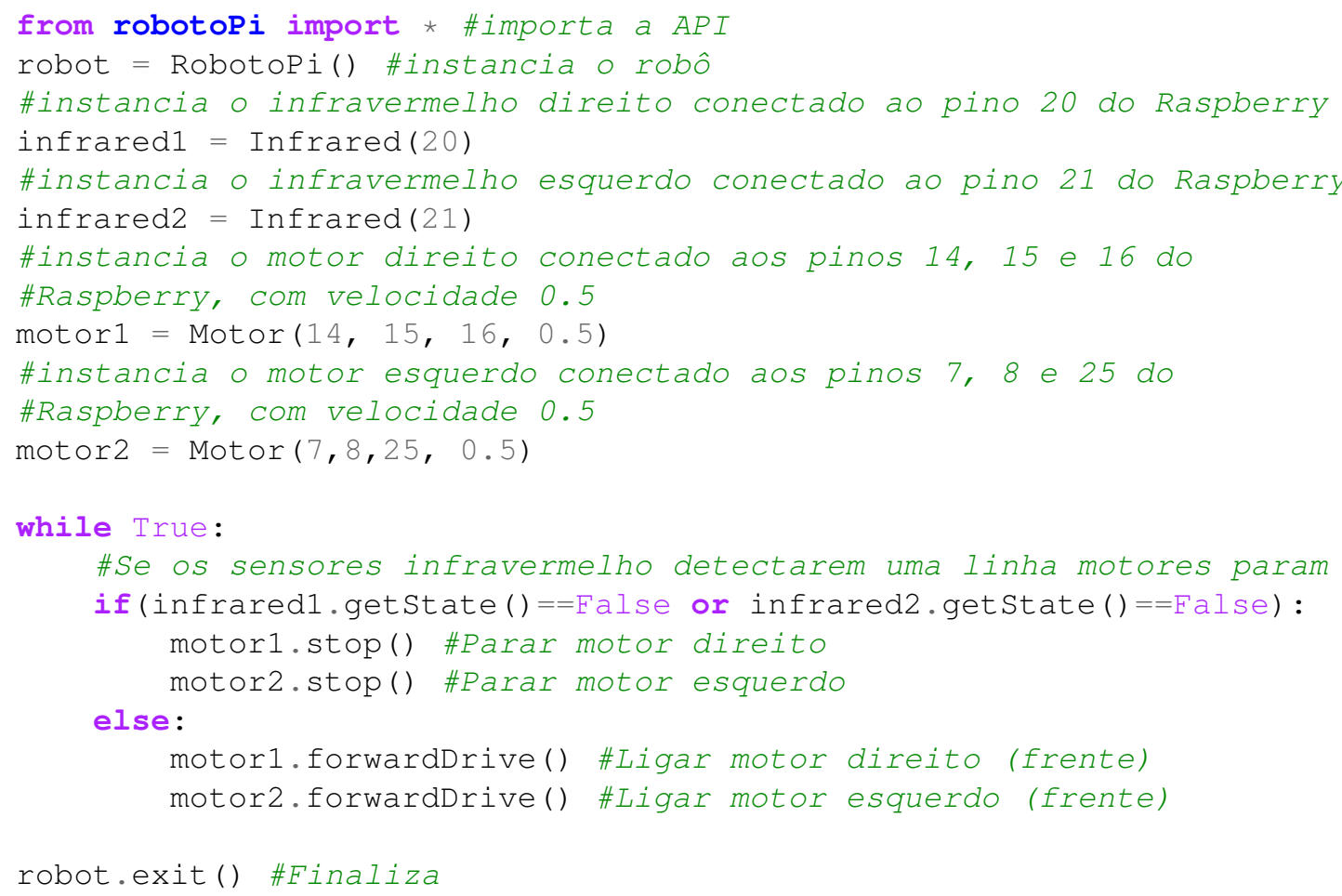

Algoritmo 1: Exemplo de utilização da API do Roboto Pi utilizando sensores infravermelhos e motores para movimentação.

\subsection{Web Site}

Para apresentação do Roboto Pi, criamos uma página web: https://robotopi. 000 webhostapp.com/. A página web tem o objetivo de disponibilizar aos usuários 
VII Congresso Brasileiro de Informática na Educação (CBIE 2018)

Anais do XXIX Simpósio Brasileiro de Informática na Educação (SBIE 2018)

acesso às informações da plataforma. A página foi dividida em seis partes: descrição do kit, documentação (manuais), código de exemplo, vídeos, e informações de contato. $\mathrm{Na}$ descrição é feita uma breve explanação da proposta da plataforma. A documentação refere-se aos manuais que visam auxiliar na utilização do kit, bem como a sugestão de atividades. Na seção exemplo são disponibilizados códigos que exemplificam como utilizar alguns sensores como forma de apresentação, visto que, na seção de API Scripting há uma quantidade maior de exemplos. Os vídeos possuem o objetivo de complementar o material pertinente a plataforma como uma opção de utilização. E a seção de informações, que disponibiliza o mantedor do projeto e contato.

\section{Considerações Finais}

Esse artigo apresentou o desenvolvimento de um kit de RP chamado Roboto Pi, destinado ao ensino introdutório de programação de estudantes de cursos técnicos e superiores. A composição do hardware e software possui como foco abstrair conceitos de eletrônica básica, não referentes à disciplina. Além disso, desenvolvemos uma API Python que facilita a compreensão em relação a utilização dos sensores, bem como materiais complementares em forma de manuais. O manual do aluno visa propiciar a compreensão do funcionamento básico da API em relação aos sensores. Já o manual do professor disponibiliza planos de aula com o objetivo de auxiliar a aplicação do Roboto Pi em disciplinas de introdução à programação.

Como trabalhos futuros, $\mathrm{O}$ Roboto Pi passará por testes para validar sua eficiência no auxílio ao ensino de programação em nível introdutório. Realizando um estudo de caso, observando duas turmas de nível superior e técnico. Ministrando aulas utilizando como ferramenta o Roboto Pi em uma das turmas para cada nível, avaliar se a aprendizagem foi superior a turma que não utilizou o kit robótico e/ou se houve mais desenvoltura dos alunos no aprendizado do conteúdo ministrado. A partir do uso do kit, serão realizados estudos para verificar se os professores gostaram de utilizar o Roboto Pi em sala de aula e quais as criticas ao kit. O Roboto Pi também passará por adequações para estender a compatibilidade da API para mais sensores. Será implementado outra API para integração com linguagem de programação visual no intuito de expandir o público alvo para crianças e adolescentes. Com isso, será necessário desenvolver outros materiais didáticos específicos.

\section{Referências}

Adafruit (2018). Adafruit webide. https://learn.adafruit.com/webide/overview.

Becker, F. (2008). Modelos pedagógicos e modelos epistemológicos. Metodologia: construção de uma proposta científica. Curitiba, Camões, pages 45-56.

Blank, D., Kumar, D., Meeden, L., and Yanco, H. (2004). Pyro: A python-based versatile programming environment for teaching robotics. Journal on Educational Resources in Computing (JERIC).

de Robótica, O. B. (2017). Regras da final nacional modalidade prática. http://www.obr.org.br/modalidade-pratica/etapa-nacional/. Disponível em: 25 de março de 2018. 
VII Congresso Brasileiro de Informática na Educação (CBIE 2018)

Anais do XXIX Simpósio Brasileiro de Informática na Educação (SBIE 2018)

Devmedia (2018). Criando apis de desenvolvimento de software. https://www.devmedia.com.br/criando-apis-de-desenvolvimento-de-software/30445.

Disponível em: 25 de março de 2018.

Fischertechnik (2018). Fischertechnik robo tx explorer manual. http://www.fischertechnik.com.mx/pdfs/519341.pdf. Disponível em: 21 de março de 2018.

Foundation, R. P. (2018). Raspberry pi. https://www.raspberrypi.org/.

Giraffa, L. and Mora, M. (2016). Evasão na disciplina de algoritmo e programação: um estudo a partir dos fatores intervenientes na perspectiva do aluno. Congresos CLABES.

Gomes, A., Henriques, J., and Mendes, A. J. (2008). Uma proposta para ajudar alunos com dificuldades na aprendizagem inicial de programação de computadores. In Educação Formação e Tecnologias.

Gomes, G., Martinho, J., Bernardo, M., Matos, F., and Abrantes, P. (2012). Dificuldades na aprendizagem da programação no ensino profissional. II Congresso Internacional TIC e Educação.

Kato, L., Braga, R., and Pazmino, A. V. (2015). Kit didático para ensino de robótica. Blucher Design Proceedings 2.3.

LEGO (2018). Lego mindstorms ev3 manual. https://goo.gl/h1N81d.

Marques, M. N. et al. (2014). Sistema de controle de navegação e comunicação para um robô móvel autônomo baseado na tecnologia raspberry pi.

Miller, J. A. (2004). Promoting computer literacy through programming Python. University of Michigan.

Miranda, L. D., Sampaio, F., and Borges, J. A. S. (2010). RoboFácil: Especificação e Implementação de um Kit de Robótica para a Realidade Educacional Brasileira. Brazilian Journal of Computers.

Neves, R. d. A. and Damiani, M. F. (2006). Vygotsky e as teorias da aprendizagem.

of Fritzing foundation, F. (2018). Fritzing. http://fritzing.org.

Pete (2018). Alpha maker. https://www.pete.com.br/robotica-com-kit-alpha-maker. Disponível em: 21 de março de 2018.

Rocha, R. (2006). Utilização da robótica pedagógica no processo de ensino aprendizagem de programação de computadores. Centro Federal e Educação Tecnológica de Minas Gerais.

Roosendaal, T. (2007). The essential Blender: guide to 3D creation with the open source suite Blender. Blender Foundation.

Silva, S. R. X. d. (2017). Protótipo de um robô móvel de baixo custo para uso interdisciplinar em cursos superiores de engenharia e computação.

Zanetti, H. and Oliveira, C. (2016). Práticas de ensino de Programação de Computadores com Robótica Pedagógica e aplicação de Pensamento Computacional. V Congresso Brasileiro de Informática na Educação (CBIE 2016). 